\title{
Comparative Evaluation of the Efficacy of Laser Therapy and Fibroblastic Growth Factor Injection on Mucosal Wound Healing in Rat Experimental Model
}

\author{
Maryam Allameh ${ }^{1 *}$, Saeedeh Khalesi ${ }^{2}$, Faezeh Khozeimeh', Elham Faghihian ${ }^{3}$ \\ ${ }^{1}$ Dental Research Center, Department of Oral Medicine, Isfahan University of Medical Sciences, Isfahan, Iran \\ ${ }^{2}$ Dental Material Research Center, Department of Oral and Maxillofacial Pathology, Isfahan University of Medical Sciences, \\ Isfahan, Iran \\ ${ }^{3}$ Isfahan University of Medical Sciences, Isfahan, Iran
}

\author{
*Correspondence to \\ Maryam Allameh, Dental \\ Research Center, Department \\ of Oral Medicine, Isfahan \\ University of Medical Sciences, \\ Isfahan, Iran \\ Tel: +989131860713 \\ Email: maryamallameh@dnt. \\ mui.ac.ir
}

Published online 28 July 2018

\begin{abstract}
Introduction: The aim of the present study was to compare the effects of laser and basic fibroblastic growth factor (bFGF) treatment on operative wound healing in a rat model.

Methods: Sixty-six male Wistar rats were employed in this study. A 10-mm surgical wound was created on the buccal mucosa of each rat, under anesthesia, and then the rats were divided into 3 groups of 22: (1) GF group (received subcutaneous injection of bFGF), (2) laser group (treated with low-level laser irradiation), and (3) control group (received no treatment). On day 5, half of the rats in each group and on day 10 the other half, were sacrificed. Afterward, samples were taken from rats' buccal mucosa for histological assay and scoring. The data were analyzed using MannWhitney test $(\alpha=5 \%)$.

Results: On day 5 there was not any significant difference between GF and control groups; however, the laser group showed clinically delayed wound coverage, compared to other groups $(P<0.05)$. On day 10, histological examination demonstrated marked vascular granulation tissue ( GT) in GF group. Collagen production was significantly prominent in laser group compared to GF treated samples $(P=0.004)$. Inflammation of GT in GF and laser groups was significantly less than that in control samples $(P=0.005$ and $P=0.001$, respectively).

Conclusion: The components of wound matrix induced by GF and laser treatment were significantly different. Although bFGF or laser treatment of oral wounds, under the conditions of the present study, did not accelerate wound healing, they showed some other notable effects on the quality of healing.

Keywords: bFGF; Granulation tissue; Laser; Oral; Wound healing.
\end{abstract}

\section{Introduction}

Mouth sores are common ailments that appear on any site of the oral cavity, including the lips, cheeks, gums, tongue, floor, and roof of the mouth. Most ulcers are benign and resolve spontaneously. ${ }^{1}$ These lesions can arise as a result of a vast number of factors, including local causes (e.g. chemical, mechanical and thermal injuries; infection; neoplasia; ischemia; radiation), systemic conditions (autoimmune and inflammatory disorders, side effects of various drugs, systemic infections, hematologic status or inherited conditions) $)^{2}$ or they may be of an unknown cause like idiopathic aphthous stomatitis. ${ }^{3}$

Mouth ulcers can be painful when eating, drinking or brushing teeth. ${ }^{4}$ On the other hand, the mouth is the first barrier against foreign bodies and microorganisms, ${ }^{5}$ which can be compromised by wounds.
The wound healing process is a dynamic one which can be divided into three phases: inflammatory, proliferation, and maturation. During the first stage, clot is formed and eventually, vessels dilate to allow essential cells and agents to reach the wounded area. The proliferation phase is characterized by the formation of granulation tissue (GT) and also new vessels. The surface of the wound is then covered with epithelium. In the last stage (maturation), remodeling of collagen would occur. ${ }^{6}$ The structure and composition of GT in the proliferation stage is often the indicator of how the wound healing process would go on. Better perfusion of tissue with new vessels would result in promotion of healing; on the other hand, fibroblasts and collagen formation have also a key role in the healing process. $^{7,8}$

In spite of the fact that our understanding of wound

Please cite this article as follows: Allameh M, Khalesi S, Khozeimeh F, Faghihian E. Comparative evaluation of the efficacy of laser therapy and fibroblastic growth factor injection on mucosal wound healing in rat experimental model. J Lasers Med Sci. 2018;9(3):194-199. doi:10.15171/jlms.2018.35. 
healing has vastly increased over the last decade, unfortunately, there is not a universally approved treatment for oral and cutaneous wounds. ${ }^{9}$ The best treatment is the modality which would both accelerates the healing process and relieves pain. For pain relief of oral ulcers, practitioners usually prescribe a manually made mouth wash that includes dexamethasone or betamethasone and diphenhydramine or lidocaine in addition to nystatin drop. ${ }^{10}$ This cocktail would only alleviate patient symptoms, with no effect on the healing process. ${ }^{7,11}$

In previous studies, several modalities have been introduced for acceleration of wound healing, such as traditional medicine $e^{12,13}$ and chemical drugs like phenytoin. ${ }^{14}$

Laser therapy, introduced in 1976 for wound healing, ${ }^{15}$ is proposed not only as a modality to relieve pain ${ }^{16}$ but also for its healing promotion effects. ${ }^{14,17,18}$

The exact mechanism underlying laser efficacy in accelerated wound healing has not been demonstrated yet. ${ }^{19}$ In the past, the terms 'photobioactivation' and 'biostimulation' were frequently used based on the stimulatory effects, later replaced by 'biomodulation', because inhibitory effects were also noted. ${ }^{20}$ There are contradictory statements on the efficacy of laser on wound healing; this is largely due to different devices and laser parameters applied in these studies as well as the variety of study models, including animal and human, which make the comparison difficult. Lucas et $\mathrm{al}^{20}$ found that animal experiments and clinical studies that address the biological effects of LLLT on wound healing have been conducted simultaneously, rather than in sequence.

Yasukawa et $\mathrm{al}^{21}$ investigated the effect of a He-Ne laser on operative wound healing in rats. They histologically examined various laser parameters on its biologic impacts and showed that with higher laser power and considering intervals between laser sessions, a more desirable healing process can be achieved.

Up to now, many studies have examined different growth factors on wound healing, such as epidermal growth factor (EGF), transforming growth factor (TGF $\beta^{22}$,) recombinant platelet-derived growth factor (rPDGF ${ }^{23,24}$ and basic fibroblastic growth factor (bFGF). ${ }^{25}$

$\mathrm{bFGF}$ is a recognized potent stimulator of endothelial cells and neo-vessel formation. ${ }^{18}$ This agent has shown promising effects in healing promotion ${ }^{24,25}$; however, there is no evidence on its efficacy on mucosal wounds. Therefore, the present study was conducted to compare healing promotion effects of low-level laser therapy and fibroblastic growth factor injection on mucosal wounds, clinically as well as microscopically, in rat experimental models.

\section{Methods}

Animals

Sixty-six male Wistar rats (each group consisting of 22 rats) aged 8 weeks (average body weight of 230 g) were used in this study.

Rat Buccal Mucosal Wound Model

A wound with a length of $10 \mathrm{~mm}$, width of $5 \mathrm{~mm}$ and depth of $5 \mathrm{~mm}$ was created with scalpel in the left buccal mucosa of all experimental rats under anesthesia with isoflurane (by means of inhalation). The wounds were not sutured so they were supposed to heal by secondary intention. To control the bleeding, intensive pressure with sterile gauze was used. Moreover, enrofloxacin (Sigma Aldrich, USA) intra-muscular injection was used at $5 \mathrm{mg} /$ $\mathrm{kg}$, once a day for 3 days to prevent infection.

\section{Laser Therapy Protocol}

A diode laser (Fionoe, Jiangsu, China), with parameters summarized in Table 1, was used for laser-assisted treatment of experimental wounds in the laser group. This procedure was carried out after superficial inhalation sedation for each rat and opening of its mouth with a molt mouth gag (Figure 1). The sequence of irradiation was every other day (first, third and fifth day after the operation).

\section{Treatment by Growth Factor}

One milliliter of bFGF (Sigma Aldrich, USA) with a concentration of $0.1 \mu \mathrm{g} / \mathrm{mL}$ was injected submucosally in the bed of the wounds every other day (first, third and fifth day after the operation).

Table 1. Laser Parameters and Specifications

\begin{tabular}{ll}
\hline Parameter & Explanation \\
\hline Type & Diode: GaAlAs \\
Wavelength & $810 \mathrm{~nm}$ \\
Cross section of laser tip & $1 \mathrm{~cm}^{2}$ \\
Power & $0.1 \mathrm{~W}$ \\
Intensity & $0.1 \mathrm{~W} / \mathrm{cm}^{2}$ \\
Dose & $4 \mathrm{~J} / \mathrm{cm}^{2}$ \\
Irradiation time & $40 \mathrm{~s}$ \\
Irradiation mode & $\mathrm{CW}$ \\
Distance from area & In Contact \\
\hline
\end{tabular}

Abbreviation: CW, continuous wave.

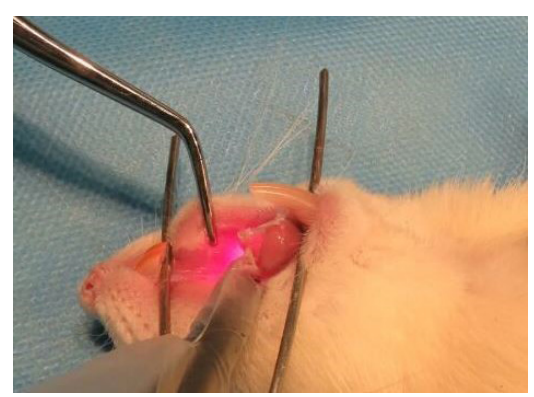

Figure 1. Laser Application in Rat Experimental Buccal Wound. 


\section{Clinical Assessment of Wound Closure}

The edge of each wound was traced on to a glass slide and the wound area was determined by quantifying the surface area of an open wound. The trace taken immediately after the operation was used as the reference or original area and all further areas were recorded as a percentage of the original area. Wound closure percentage was calculated as follows:

$\%$ wound closure $=\frac{(\text { area on day } 0-\text { open area on final day })}{\text { areaonday } 0} \times 100$

Preparation of Mucosal Specimens

On the fifth day after the operation, half of the animals in each group, and on day 10, the other half were sacrificed with an intraperitoneal overdose of chloroform. Afterwards, rectangular specimens (each with a width of $10 \mathrm{~mm}$ and length of $30 \mathrm{~mm}$ ) were removed from the operation site of each rat and were fixed in $10 \%$ formalin solution. The specimens were paraffin embedded and the samples were sectioned in thin slices $(5 \mu \mathrm{m})$ for slide preparation. Finally, hematoxylin and eosin (H\&E) staining was carried out for histopathological assessment under a light microscope.

Histological Examination

The scoring of the samples was done by a blinded pathologist, similar to the histological scoring system used in the study by Taheri et $\mathrm{al}^{14}$ with some minor modifications (Table 2).

\section{Statistical analyses}

Analysis of the histological and clinical rankings was performed using the Kruskal-Wallis test with individual comparisons performed by Mann-Whitney test $(\mathrm{p}<0.05)$. The analysis was performed using the SPSS 23 package.

\section{Results}

Day 5

In the control group, $63.3 \%$ of the cases showed less than $50 \%$ epithelial migration, and PMN infiltration was moderate to marked in more than $80 \%$ of them (Figure 2 ). Mean reduction in wound size in GF and control groups was significantly larger than that in the laser group. This result was also demonstrated in microscopic view where wound coverage with epithelium in the group treated with GF was significantly larger than that in the laser group. Although GF group received higher mean scores of parameters indicative of wound healing promotion

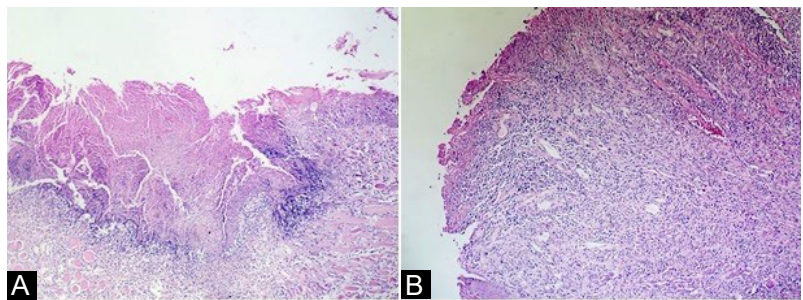

Figure 2. A, B. Histological Findings 5 Days After Wounding in Control Group. There is not any epithelial coverage on the wound (thickness of cut edges) and the GT is filled with PMNs (H\&E; $\times 40)$.

than the control group, this difference was not statistically significant (Figure 3). $P$ values are given in Table 3.

Similarly, the highest scores of collagen production belonged to samples of the laser treated group, although the difference was not significant (Figure 4). On the other hand, wound closure percentage of laser group was significantly smaller than the control group $(P=0.034)$.

\section{Day 10}

Clinical assay of wound closure percentage on day 10 showed more than $70 \%$ reduction in size of the open area in the group treated with subcutaneous injection of bFGF. Clinical wound closure and microscopic epithelial wound coverage were significantly accelerated in GF group compared to laser-treated cases. Furthermore, GF group showed markedly augmented vascular formations compared to laser and control groups (Figure 5). It was observed that laser induced significantly greater collagen synthesis than bFGF (Figure 6).

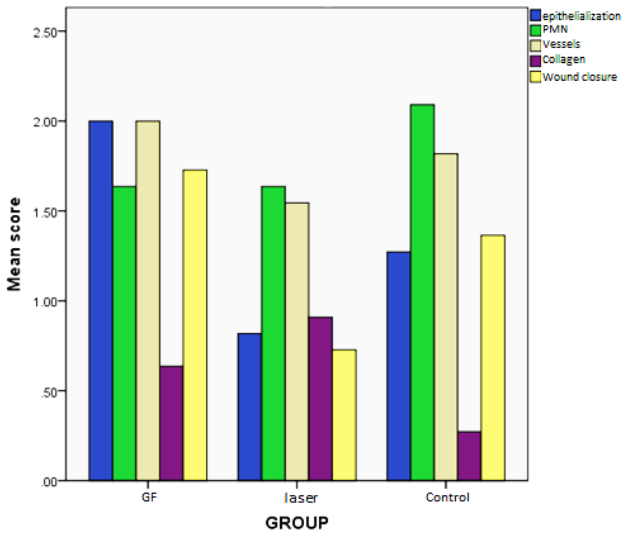

Figure 3. Day 5 Mean Scores of Each Measured Parameter in Separate Groups.

Table 2. Scores of Each Clinical and Histological Paramete

\begin{tabular}{|c|c|c|c|c|c|}
\hline Score & Epithelialization & PMNs & Collagen & Vessels & Clinical Wound Closure Percent \\
\hline 0 & The thickness of cut edges & Absent & Absent & Absent & No reduction \\
\hline 1 & Migration of cells $(<50 \%)$ & Mild & Mild & Mild & Less than $50 \%$ \\
\hline 2 & Migration of cells ( $>50 \%$ ) & Moderate & Moderate & Moderate & More than $50 \%$ \\
\hline 3 & Bridging the excision & Marked & Marked & Marked & Almost $100 \%$ \\
\hline
\end{tabular}


Table 3. $P$ Values of Differences in Pathological Scores of Groups Compared

\begin{tabular}{|c|c|c|c|c|c|c|}
\hline \multirow{2}{*}{ Groups } & & \multicolumn{5}{|c|}{ Examined Pathological Indices } \\
\hline & & Wound Size & Epithelial Coverage & Inflammation & Collagen & Vascular Formation \\
\hline \multirow{3}{*}{ GF day 5} & LLLT day 5 & $0.001^{*}$ & $0.005^{*}$ & 0.847 & 0.478 & 0.217 \\
\hline & Control day 5 & 0.151 & 0.056 & 0.171 & 0.243 & 0.056 \\
\hline & GF day 10 & $0.04 *$ & $0.001^{*}$ & $0.013^{*}$ & 0.133 & 0.076 \\
\hline \multirow[t]{2}{*}{ LLLT day 5} & C day 5 & $0.034^{*}$ & 0.243 & 0.365 & 0.088 & 0.478 \\
\hline & GF day 10 & $0.001^{*}$ & $0.000^{*}$ & 0.652 & $0.004^{*}$ & $0.000^{*}$ \\
\hline \multirow[t]{2}{*}{ LLLT day 10} & C day 10 & $0.002 *$ & $0.028^{*}$ & $0.001^{*}$ & 0.133 & 0.365 \\
\hline & LLLT day 5 & 0.217 & $0.019 *$ & $0.016^{*}$ & $0.005^{*}$ & 0.133 \\
\hline GF day 10 & C day 10 & 0.193 & 0.088 & $0.005^{*}$ & 0.3 & $0.002 *$ \\
\hline
\end{tabular}

C, control.

* Significant difference.

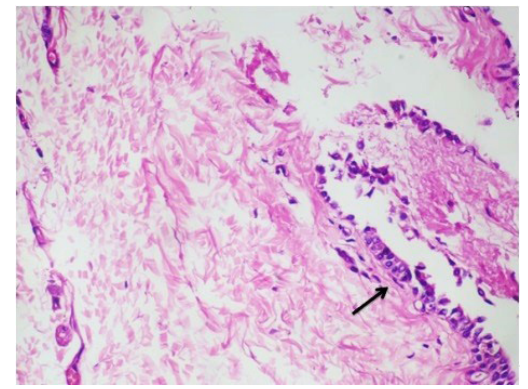

Figure 4. Collagen Deposition in GT in a Laser-Treated Wound on Day 10 of the Experiment. Note the inconsistent epithelium (arrow) $(\mathrm{H} \& \mathrm{E} ; \times 40)$

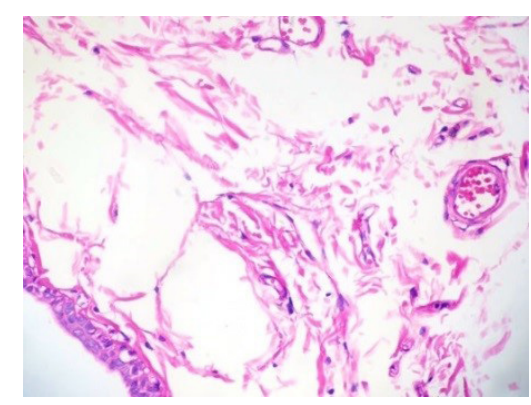

Figure 5. Histologic View of GT 10 Days After Wounding in a Sample Treated With bFGF. High vascular GT and a complete coverage of epithelium are evident $(\mathrm{H} \& \mathrm{E} ; \times 100)$

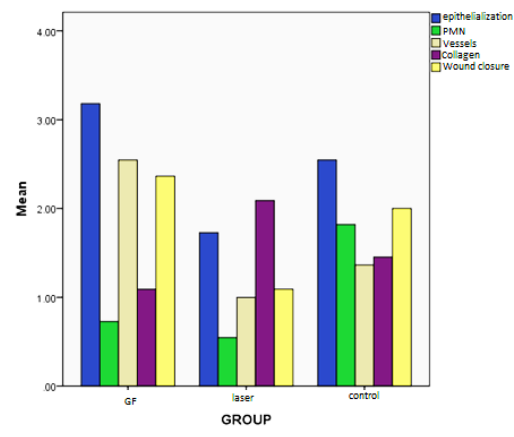

Figure 6. Day10 Mean Scores of Each Measured Parameter in Separate Groups.
Inflammatory cell infiltration was a predominant phenomenon in most wounds in control group. However, this feature was significantly less observed in GF and laser groups (Figure 2).

\section{Discussion}

The GT color and condition can foreshow the wound healing process. ${ }^{23}$ Thick GT with dominance of vessels was observed in most samples of GF group. This result was consistent with previous investigations on bFGF application, which showed accumulation of only provisional matrix (filled with neovessels) after bFGF injection into wounds. ${ }^{23}$ Moreover, in the present investigation, the bFGF treated group showed a higher percentage of wound closure compared to the laser and control groups. This can be related to the effect of bFGF on endothelial cells and proliferation of vessels which cause a bloody bed in the wound. The drainage of epithelium would make wound coverage faster. It should be investigated in future studies if the strength of scar tissue after complete healing is adequate enough with such treatment modality. Nevertheless, wound coverage in GF group (clinically and microscopically) did not statistically supersede the control group, which may be attributed to the low number of samples in each group. However, in Pierce et al's examination, ${ }^{23}$ bFGF injection was followed by accelerated wound healing. This confliction may be justified by the difference in the site of operation, which was skin in the aforementioned study; Possibly, because the skin contains a lower number of vessels than mucosal tissues, ${ }^{26}$ bFGF prescription could play a valuable role in augmentation of vascular formations and cause a significant difference between bFGF and control groups. Oda et $\mathrm{al}^{27}$ investigated bFGF biological mechanism in rat palatal wound healing; they found higher levels of fibroblast growth factor receptor 1 (FGFR1)-positive cells in the group treated by single dose topical bFGF. So stimulated cell proliferation was reported as the mechanism of bFGF action in wound healing. However, stimulation of collagen maturation was also reported in palatal wounds treated with bFGF; which is in confliction 
with the results of the present investigation. This may be due to the methodological differences between these two studies, as Oda et $\mathrm{al}^{27}$ investigated cellular and subcellular effects of this modality, whereas in the present study only microscopic view of tissues was examined and it turns out that many cellular interactions would be overlooked.

There are a large number of studies which demonstrated healing promotion effects for laser. ${ }^{28-30}$ The mechanism of low-level laser therapy in healing promotion is not completely understood yet; however, it is supposed that laser efficacy is not only due to its heating effects but rather to its photochemical, photophysical and/ or photobiological effects; many name these as photobiomodulation. It seems that laser illumination can improve local circulation, cell proliferation, and collagen synthesis by increasing cell access to ATP. ${ }^{31}$ However, similar to in vitro and in vivo surveys of Solmaz et al, ${ }^{8}$ the present study showed that laser induced a delayed healing process of oral wounds in rats. This confliction might possibly be attributed to different laser parameters, the difference between the employed methods (such as the site of the wound, animal or human study, scales to measure, healing stage) and laser parameters. For example, in the study of Tabakoglu et al, ${ }^{28}$ the wound size was half the size of the ulcer examined in the present study; therefore, it is predicted that wound closure in that study was more due to the contraction mechanism rather than healing by secondary intention; and as the wounds in laser group have higher amounts of collagen, the susceptibility to contract is higher in this group than control group. ${ }^{32}$ Collagen fibers in laser group showed considerably higher levels than GF group. This characteristic of laser therapy was previously reported by Taheri et $\mathrm{al},{ }^{14}$ which resulted in a more firm tissue despite increasing the risk of keloid scar tissue creation. Yet, laser-induced collagen production did not differ significantly with the amount of collagen accumulated normally in the same stage of wound healing in control group; this result might become significant if a larger number of cases are employed. Inhibition of collagen aggregation caused by bFGF has already been reported in the literature; this effect is in line with making penetration of neovessels to tissues easy and would last until the latest phase of healing. ${ }^{23}$ According to the results of the present study, it is advisable to use bFGF injections in earlier phases of wound healing to achieve vascular GT which facilitates wound closure, and employ laser therapy in later stages to induce collagen production and induce GT maturation.

Both bFGF and laser treatments reduced inflammation considerably in the final phase of healing. This may be attributed to the reported pain reduction capabilities of laser therapy on wounds. ${ }^{33}$

\section{Conclusion}

The results showed that bFGF injection of oral wounds did not lead to a faster wound closure, and laser therapy caused a delay in this process. bFGF induced GT was more vascular; in contrast, laser caused maturated GT filled with collagen bundles.

\section{Ethical Considerations}

The experiment was conducted in compliance with the protocol approved by the Institutional Animal Care and Use Committee of Esfahan University of Medical Sciences.

\section{Conflict of Interests}

The authors declare that they have no competing interests.

\section{References}

1. Paleri V, Staines K, Sloan P, Douglas A, Wilson J. Evaluation of oral ulceration in primary care. BMJ. 2010;340:c2639. doi:10.1136/bmj.c2639

2. Siu A, Landon K, Ramos DM. Differential diagnosis and management of oral ulcers. Semin Cutan Med Surg. 2015;34(4):171-177. doi:10.12788/j.sder.2015.0170

3. Edgar NR, Saleh D, Miller RA. Recurrent Aphthous Stomatitis: A Review. J Clin Aesthet Dermatol. 2017;10(3):26-36

4. Mortazavi H, Safi Y, Baharvand M, Rahmani S. Diagnostic Features of Common Oral Ulcerative Lesions: An Updated Decision Tree. Int J Dent. 2016;2016:7278925. doi:10.1155/2016/7278925

5. Janeway CA Jr, Travers P, Walport M, Shlomchik MJ. Immunobiology: The Immune System in Health and Disease. 5th ed. New York: Garland Science; 2001.

6. Rocha Junior AM, Vieira BJ, de Andrade LC, Aarestrup FM. Effects of low-level laser therapy on the progress of wound healing in humans: the contribution of in vitro and in vivo experimental studies. J Vasc Bras. 2007;6(3):258266. doi:10.1590/S1677-54492007000300009

7. Cohen IK, Diegelmann RF, Johnson ML. Effect of corticosteroids on collagen synthesis. Surgery. 1977;82(1):15-20.

8. Solmaz H, Ulgen Y, Gulsoy M. Photobiomodulation of wound healing via visible and infrared laser irradiation. Lasers Med Sci. 2017;32(4):903-910. doi:10.1007/s10103017-2191-0

9. Rajendran R. Shafer's Textbook of Oral Pathology. [S.l.]: Reed Elsevier; 2010.

10. Little JW. Little And Falace's Dental Management Of The Medically Compromised Patient. [S.1.]: Mosby; 2017.

11. Drugs that delay wound healing. Prescrire Int. 2013;22(137):94-98.

12. Paocharoen V. The efficacy and side effects of oral Centella asiatica extract for wound healing promotion in diabetic wound patients. J Med Assoc Thai. 2010;93 Suppl 7:S166170.

13. Samarghandian S, Farkhondeh T, Samini F. Honey and Health: A Review of Recent Clinical Research. Pharmacognosy Res. 2017;9(2):121-127. doi:10.4103/09748490.204647

14. Beigom Taheri J, Bagheri F, Mojahedi M, et al. Comparison of the Effect of Low-Level Laser and Phenytoin Therapy on Skin Wound Healing in Rats. J Lasers Med Sci. 2015;6(3):124-128. doi:10.15171/jlms.2015.06

15. Mester E, Nagylucskay S, Doklen A, Tisza S. Laser 
stimulation of wound healing. Acta Chir Acad Sci Hung. 1976;17(1):49-55.

16. Aggarwal H, Singh MP, Nahar P, Mathur H, Gv S. Efficacy of low-level laser therapy in treatment of recurrent aphthous ulcers - a sham controlled, split mouth follow up study. J Clin Diagn Res. 2014;8(2):218-221. doi:10.7860/ jcdr/2014/7639.4064

17. Simunovic Z, Ivankovich AD, Depolo A. Wound healing of animal and human body sport and traffic accident injuries using low-level laser therapy treatment: a randomized clinical study of seventy-four patients with control group. J Clin Laser Med Surg. 2000;18(2):67-73. doi:10.1089/ clm.2000.18.67

18. Hopkins JT, McLoda TA, Seegmiller JG, David Baxter G. Low-Level Laser Therapy Facilitates Superficial Wound Healing in Humans: A Triple-Blind, Sham-Controlled Study. J Athl Train. 2004;39(3):223-229.

19. Posten W, Wrone DA, Dover JS, Arndt KA, Silapunt S, Alam M. Low-level laser therapy for wound healing: mechanism and efficacy. Dermatol Surg. 2005;31(3):334-340.

20. Lucas C, Criens-Poublon LJ, Cockrell CT, de Haan RJ. Wound healing in cell studies and animal model experiments by Low Level Laser Therapy; were clinical studies justified? a systematic review. Lasers Med Sci. 2002;17(2):110-134.

21. Yasukawa A, Hrui H, Koyama Y, Nagai M, Takakuda K. The effect of low reactive-level laser therapy (LLLT) with helium-neon laser on operative wound healing in a rat model. J Vet Med Sci. 2007;69(8):799-806.

22. Roberts AB. Transforming growth factor-beta: activity and efficacy in animal models of wound healing. Wound Repair Regen. 1995;3(4):408-418. doi:10.1046/j.1524475X.1995.30405.x

23. Pierce GF, Tarpley JE, Yanagihara D, Mustoe TA, Fox $\mathrm{GM}$, Thomason A. Platelet-derived growth factor (BB homodimer), transforming growth factor-beta 1 , and basic fibroblast growth factor in dermal wound healing. Neovessel and matrix formation and cessation of repair. Am J Pathol. 1992;140(6):1375-1388.
24. Greenhalgh DG, Sprugel KH, Murray MJ, Ross R. PDGF and FGF stimulate wound healing in the genetically diabetic mouse. Am J Pathol. 1990;136(6):1235-1246.

25. Fujisawa K, Miyamoto Y, Nagayama M. Basic fibroblast growth factor and epidermal growth factor reverse impaired ulcer healing of the rabbit oral mucosa. J Oral Pathol Med. 2003;32(6):358-366.

26. Szpaderska AM, Zuckerman JD, DiPietro LA. Differential injury responses in oral mucosal and cutaneous wounds. J Dent Res. 2003;82(8):621-626. doi:10.1177/154405910308200810

27. Oda Y, Kagami H, Ueda M. Accelerating effects of basic fibroblast growth factor on wound healing of rat palatal mucosa. J Oral Maxillofac Surg. 2004;62(1):73-80.

28. Tabakoglu HO, Sani MM, Uba AI, Abdullahi UA. Assessment of circular wound healing in rats after exposure to 808-nm laser pulses during specific healing phases. Lasers Surg Med. 2016;48(4):409-415. doi:10.1002/ lsm. 22462

29. Fulop AM, Dhimmer S, Deluca JR, et al. A meta-analysis of the efficacy of phototherapy in tissue repair. Photomed Laser Surg. 2009;27(5):695-702. doi:10.1089/pho.2009.2550

30. Woodruff LD, Bounkeo JM, Brannon WM, et al. The efficacy of laser therapy in wound repair: a meta-analysis of the literature. Photomed Laser Surg. 2004;22(3):241-247. doi:10.1089/1549541041438623

31. Lins RD, Dantas EM, Lucena KC, Catao MH, GranvilleGarcia AF, Carvalho Neto LG. Biostimulation effects of low-power laser in the repair process. An Bras Dermatol. 2010;85(6):849-855.

32. Berry DP, Harding KG, Stanton MR, Jasani B, Ehrlich HP. Human wound contraction: collagen organization, fibroblasts, and myofibroblasts. Plast Reconstr Surg. 1998;102(1):124-131; discussion 132-124.

33. Suter VGA, Sjolund S, Bornstein MM. Effect of laser on pain relief and wound healing of recurrent aphthous stomatitis: a systematic review. Lasers Med Sci. 2017;32(4):953-963. doi:10.1007/s10103-017-2184-Z 